PAR T I

CLASSIFICATION OF SLIT SPECTRA 


\title{
THE ROLE OF CLASSIFICATION OF SLIT SPECTROGRAMS
}

\author{
PH. C. KEENAN
}

Perkins Observatory, The Ohio State and Ohio Wesleyan Universities, Ohio, U.S.A.

I take it as evident that the aims of stellar classification are the same whether we work by direct photoelectric photometry through filters, by spectrophotometry, or by visual classification of spectrograms. In all cases we are trying to make direct estimates of as many physical properties of the stars as possible, and as nearly as I can judge, no one method has such exclusive advantages that it seems likely to replace all others in the immediate future. The problems are so difficult - so many subtle differences between stars that we once thought of as identical are turning up now - that we must use every effective means of attack. The present challenge is to combine these methods most effectively.

Where spectroscopy through slits can contribute most is in the establishment of accurate standards which the photometric people can use to relate their precise readings made through various band passes to differences in particular molecular or atomic features in the spectrum. This morning I can give you only a few examples of recent refinements in classification - and must omit important contributions, such as the completion of the Córdoba Atlas by the groups headed by Dr Landi and the Jascheks.

I. Since Dr Morgan was not able to be here himself, I want to begin with the revision of the classification of A- and F-type stars that Morgan and Abt (1971) have just completed. They have kindly allowed me to summarize their results in advance of publication. The spectrograms are a very uniform set taken by Abt at $125 \AA \mathrm{mm}^{-1}$ with the small prismatic spectrograph on the 36-in. telescope at Kitt Peak. For this spectral range they consider the relatively small scale actually an advantage, for on such spectrograms the appearance of the G-band can be judged readily. I should mention that for earlier spectral classes (B-stars), Morgan and his students have been using higher dispersions $\left(20 \AA \mathrm{mm}^{-1}\right)$ to bring out fainter lines than could be used before.

For types A7-G0 and luminosity classes II to IV, Morgan and Abt established the zero points from original MK standard stars, but within that framework a real gain in precision has been attained by using as many lines as possible between $\lambda 3850$ and $\mathrm{H} \gamma$, plus the apparent structure of the G-band. They employed criteria usual for that scale and in addition took into account the general appearance of the strong features, blended and unblended; particularly the relative strength of the stronger lines of ions (Fe II, Ti II, Sr II), and of neutral atoms (Fe, Mn). This total classification is important, and I think that most experienced spectroscopists do it almost unconsciously, though it is difficult to describe the process. It is much easier to tell how one sensitive line ratio is related to, say, luminosity class. The importance of the overall appearance of the spectrum is at least twofold. First, any slight but general weakening or strength- 
ening of the absorption lines, as compared to a normal star of the type, is likely to be detected. Second, a peculiarity may be shown by some feature that is not normally used as a criterion of classification; whereas, if one just mechanically records the intensity ratios of the standard criteria, that peculiarity can easily be overlooked.

In their work, Morgan and Abt ended up with 32 standard F-stars above the main sequence. As a particular application, they then made use of these accurate standards to examine a group of $14 \delta$ Scuti stars. These are the white variable stars, presumably pulsating, with very small amplitudes, about 0.1 mag. There has been some question about their position in the HR diagram with respect to the main sequence. The authors find that all of these $\delta$ Scuti stars are either subgiants or giants - they lie above the main sequence. These luminosities, at least for the 10 variables that show no marked peculiarities in their spectra, should be reliable. Four of the stars do have their $\mathrm{H}$ and $\mathrm{K}$ lines weakened by conspicuous amounts, and the range in both $\mathrm{H}$ and $\mathrm{K}$ strengths and in luminosities leads the authors to conclude that the $\delta$ Scuti stars do not form a homogeneous group.

Morgan and Abt find also that $\Delta m_{1}$, the differences between the Strömgren metallic line indices for the $\delta$ Scuti stars and for standard stars of the same luminosity and temperature, show a rather smooth, increasingly negative, run with increasing type. But since Strömgren some years ago found $\Delta m_{1}$ to be less sensitive to metal type for F stars earlier than F4 than those of later type, the authors conclude: "... The interpretation in terms of a progressively higher metal content with advancing MKA spectral type, for the $\delta$ Scuti stars of Table II, seems doubtful".

II. For the second example of a classification problem I shall not discuss one particular investigation, but instead consider an area of the H-R diagram that has been of increasing interest. This is the region of the giant branch near types G8-K0, which is important as a locus where evolutionary tracks for stars of different ages, populations, etc., either cross or come close together. To illustrate stellar spectra in this region Figure 1 shows a set of grating spectrograms for which the original scale was in the

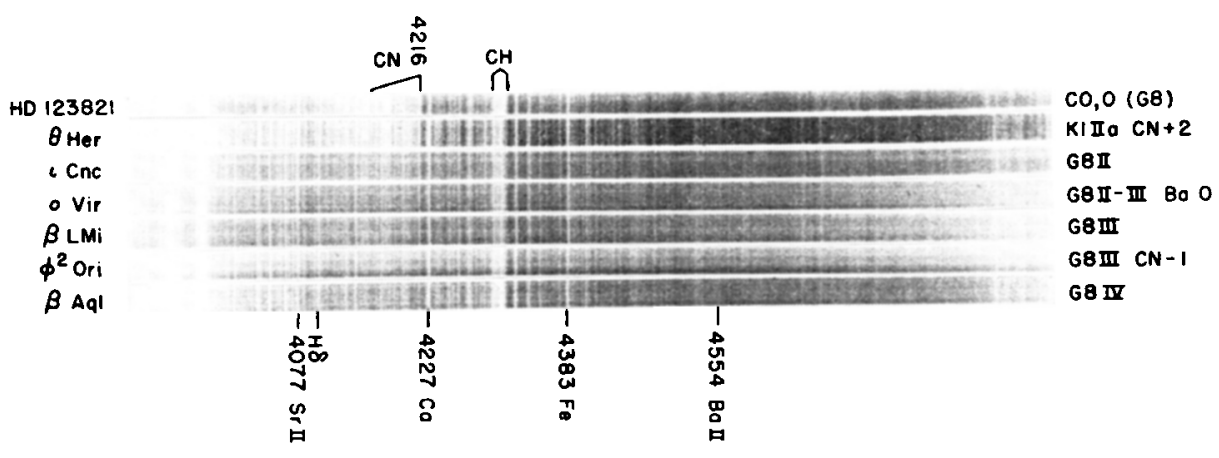

Fig. 1. Spectral peculiarities in giants, G8-K1. HD 123821 taken at Lowell Observatory with Perkins 72-inch telescope. Remaining stars taken with 32-inch reflector at Perkins Observatory. 
range from 75 to $85 \AA \mathrm{mm}^{-1}$. This is just enough greater than the scale of the original Yerkes Atlas $\left(120 \AA \mathrm{mm}^{-1}\right.$ at $\mathrm{H} \gamma$ ) to give better resolution of some of the metallic lines (e.g. Cr 4254) which are emerging through the subdivisions of type G.

Except for the top spectrum, the slide shows nothing new or very striking. I have deliberately avoided the more spectacularly peculiar objects in order to bring out instead the more subtle differences that we must be able to recognize if we hope to improve our analyses of the differences between populations.

In the illustration, the spectra of $\beta \mathrm{Aql}, \beta \mathrm{LMi}$, and $\imath \mathrm{Cnc}$ are G8III standards showing the normal luminosity effects in $\mathrm{Sr}$ II, CN, and the ratio 4376/4383. The second star from the bottom, $\phi^{2}$ Ori, is a well-known weak-CN star - a moderate population-II star. Everyone is used to looking for this peculiarity. In the opposite sense the second spectrum from the top - that of $\theta \mathrm{Her}$ - shows the opposite departure from normality. Here $\mathrm{CN}$ is unusually strong, even for a K1IIa star, and, indeed, $\theta$ Her has an intensity break at $4216 \AA$ which is the largest of any in the catalogue of Griffin and Redman (1960). Its CN absorption is about that of a normal K1 supergiant of luminosity class Iab, but if the star really were that luminous the metallic lines at $\lambda \lambda 4376$ and 4386 would appear about equal.

These stars with a positive $\mathrm{CN}$ anomaly do appear to have ratios of metals to hydrogen that are higher than normal, as given by the means of model-atmosphere analyses of abundance. The disagreements in the data for individual stars are so great relative to their means, however, that it seems an exaggeration to call them 'super metal-rich' stars (Spinrad and Taylor, 1969). It would be more realistic to refer to them merely as metal-rich, though observers who prefer to describe what they actually see in the spectra will probably continue to refer to these objects as 'strong-CN stars'.

Clearly, the luminosity classification of the strong $\mathrm{CN}$-stars must be done very carefully to avoid estimating their luminosities as too bright, just as there is the wellknown opposite tendency to underestimate the luminosities of the weak-CN stars. An interesting recent determination of statistical parallaxes by John Schmitt (1971) gave lower absolute magnitudes for G9-K4 class III stars with strong CN than for standard giants of the same types as given in Blaauw's calibration. Since, however, his spectrograms had rather low dispersion and the methods of calibration of the two groups were not identical, I mention the comparison merely to emphasize how likely we are to make systematic errors in estimating spectroscopic absolute magnitudes for stars which have even slight peculiarities in their spectra. We can minimize these errors by reviewing the general appearance of a spectrum after it has been classified by the usual criteria. Then, if $\mathrm{CN}$ or other features look abnormal, the classification is revised by means of those criteria which are least affected by the evident abnormality. This sounds obvious enough, but it is a precaution that we have all probably neglected at times.

For an instance of another and more subtle peculiarity, look at the middle spectrum ( $o$ Vir). At first glance this spectrum might be classified as G8II from the ratios $\mathrm{Sr}$ II $4077 / \mathrm{Fe} 4063,4071$ and 4216/Ca 4226. Evidently, however, the CN depression is abnormally weak for this type and the Balmer lines do not show the slight enhance- 
ment usually observed at luminosity class II as compared to a normal class III giant. The discrepancy can be largely resolved by noting the slight enhancement of $\mathrm{Ba}$ II 4554, which is normally only marginally visible in a G8 spectrum at this dispersion. The line is weaker than in most of the well-known barium stars, and $o$ Vir is accordingly classified as G8 II-III Ba0. Alternatively, one could term such objects 'semi-barium' stars. Incidentally, $o$ Vir has been recognized as a barium star by Williams (1971) also, being included in a list of 5 stars which he observed as having weak but definite enhancement of $\mathrm{Ba}$ II 4554. This classification accounts for the enhancement of $\mathrm{Sr}$ II in $o \mathrm{Vir}$, though $\mathrm{CN}$ does not show as much enhancement as is usual in barium stars. The recognition of such stars, in which the lines of the heavy elements are only slightly strengthened, offers a major challenge to either low-dispersion spectroscopy or photometry.

III. The top spectrum in this plate forms the bridge to the last group of stars that I want to discuss - for it is a newly-recognized carbon star. HD 123821 appeared in two recent lists of G-type stars with strong CN, one by McClure (1970) and one by Schmitt (1971), but the latter remarked that it was possibly a very early carbon star, with weak lines. In the illustration of an $80 \AA \mathrm{mm}^{-1}$ Flagstaff plate the 1.0 Swan band of $C_{2}$ at $\lambda 4737$ is barely visible, but it is clearly present on our $40 \AA \mathrm{mm}^{-1}$ spectrogram, leaving no doubt of the $\mathrm{C} 0$ classification. The atomic line ratios indicate a temperature equal to that of the ordinary G8 giants.

It is among the carbon stars of lower temperature that the serious problems of classification occur. When the classification using C-types was introduced (Keenan and Morgan, 1941), it was admittedly just a first approximation to a temperature sequence. Since then many attempts to estimate temperatures of the carbon stars have led to somewhat inconclusive results. Most observers have concluded that the $\mathrm{C}$-sequence represents temperatures fairly well from $\mathrm{C} 0$ through $\mathrm{C} 3$, but is not a very consistent temperature sequence in the later subdivisions. For example, WZ Cas seems definitely hotter than its original C9.1 type implied, though this star has never fitted well into any suggested sequence.

Numerous suggestions for new sequences or groupings have been made. First Bouigue (1954) and later Mrs Gordon (1971) proposed a separate class J for the stars in which a prominent band at $\lambda 6168$ shows that the $C^{13}$ isotope is unusually abundant. Richer (1971) devised a classification based on the photographic infrared in which the same symbols as in the old C-system were used, but with the numbers having entirely different significance. Gordon (1968) and others have suggested also that most of the middle C-stars form a sequence connecting directly to the Ba stars rather than to the early carbon stars. Dolidze (1969) and Kholopov (1971) at Abastumani have proposed still other subgroups based upon color-spectrum relations.

Most of the suggestions involve some good ideas that should not be forgotten, but the general picture is one of confusion. I wish that I could give you a simple solution that would make all the carbon stars fall neatly into place, but all that I can do at this time is to call your attention to some of the difficulties that stand in our way. 
Consider first that there are seven composition parameters that appear to vary within the group of carbon stars:

(1) $\mathrm{C} / \mathrm{O}$. Carbon stars are sharply defined by the condition that $\mathrm{C} / \mathrm{O}>1$, and the calculations of dissociation equilibrium by Tsuji (1964), Schadee (1968), and Greene (1971) show that only a very slight carbon excess is needed to give observable $\mathrm{C}_{2}$ bands.

(2) Metals/H. By metals we mean all the elements heavier than helium. Anomalously large values of this ratio were recognized as early as 1940 by Wurm in HD 182040 and found in other early carbon stars by Bidelman (1953).

(3) Heavy Metals/Light Metals. Enhancement of the $S$-process elements $\mathrm{Zr}, \mathrm{Y}, \mathrm{Sr}$, etc., is well known to be much greater in some carbon stars than in others.

(4) $\mathrm{C}^{13} / \mathrm{C}^{12}$. Differences in the relative strengths of the $\mathrm{C}_{2}$ bands involving the two isotopes of carbon were studied most extensively by McKellar (1949).

(5) Li/Other Metals. That the great differences in the intensity of Li I 6707 among the carbon stars represent differences in the relative abundance of lithium was confirmed by Torres-Peimbert and Wallerstein (1966) who measured [ $\mathrm{Li} / \mathrm{Ca}$ ].

(6) Tc/Other Metals. Although technetium is difficult to observe in carbon stars because the strong ultimate lines occur in the faint blue region of their spectra, its definite presence in several stars (Merrill, 1956), and absence in others (Peery, 1971) suggests marked differences in abundance.

Quite possibly we should add the abundance of nitrogen as a variable, but the difficulty of observing the $\mathrm{NH}$ bands in the $\lambda 3360$ region has kept us from having certain information on this point. If some of these abundances were tightly coupled we could simplify the problem by using only one or two abundance parameters in classification, but the available evidence suggests that they are only partially correlated; e.g., (1) with (2), and (4) with (5).

Some of the practical problems are illustrated in Figure 2, which shows a fairly typical set of red spectra of southern carbon stars. The plates were exposed to show the $\lambda 6200$ region well, since the scale of about $106 \AA \mathrm{mm}^{-1}$ was too small to permit reliable estimates of the $\mathrm{Li} 6707$ intensity except when the line is unusually strong. The top spectrum is somewhat earlier than the next three, but all have strong $\mathrm{C}_{2}$ bands. Three heads of the $\Delta v=-2$ sequence of $\mathrm{C}^{12} \mathrm{C}^{12}$ are marked at the top, and there appears to be some increase in their intensity gradient as we go from top to bottom in the set. Of the network of $\mathrm{C}^{12} \mathrm{~N}^{14}$ features which cover this whole region, only the strong heads near 5749 and $6206 \AA$ are marked.

The bottom spectrum in Figure 2 is noteworthy in showing $\lambda 6168$ and the other $\mathrm{C}^{12} \mathrm{C}^{13}$ bands very strongly, together with the depression due to $\mathrm{C}^{13} \mathrm{~N}^{14}$ near $\lambda 6260$. $\mathrm{TZ}$ Car is thus a strong $\lambda 6168$ star, and its features involving $\mathrm{C}^{13}$ are as strong as in the spectrum of any carbon star known to me. A comparable star in the northern hemisphere is T Lyr.

In spite of the obvious overlapping of bands, a rough estimate of the $C^{12} / C^{13}$ ratio can be made with the aid of the synthetic $\mathrm{CN}$ absorption spectra computed by Marenin. Figure 3 is a revision of a computer plot presented by Marenin and Greene (1971) at the August, 1971, meeting of the AAS. The absorption curves were computed for 
a simple Milne-Eddington atmosphere, using the known $f$-values for the $\mathrm{C}^{12} \mathrm{~N}^{14}$ molecule, and smoothed by giving each molecular band line a Doppler half-value width of $2.5 \AA$ to match approximately the resolution of the spectrograms of Figure 2 . The lower curve is that of a pure $\mathrm{C}^{12} \mathrm{~N}^{14}$ molecule, while the upper represents a mixture of $\mathrm{C}^{12} \mathrm{~N}^{14}$ for $\mathrm{C}^{12} / \mathrm{C}^{13}=4$. It can be seen that the model mixture predicts that the $\lambda 6206$ head of $\mathrm{C}^{12} \mathrm{~N}^{14}(4,0)$ will be slightly stronger than the corresponding $\mathrm{C}^{13} \mathrm{~N}^{14}$ feature near $6260 \AA$. In Figure 2, the $\mathrm{C}^{13} \mathrm{~N}^{14}$ feature is more conspicuous, and further computations by Miss Marenin indicate that $C^{12} / C^{13}=2$ gives a reasonably good fit for TZ Car. This ratio is lower than has generally been suggested on theoretical grounds, but an iso-intensity argument should have some weight. The near equality of the $\mathrm{C}_{2}$ heads, $\mathrm{C}^{12} \mathrm{C}^{12}$ at $6191 \AA$ and $\mathrm{C}^{12} \mathrm{C}^{13}$ at $6168 \AA$ tends to support our conclusion.

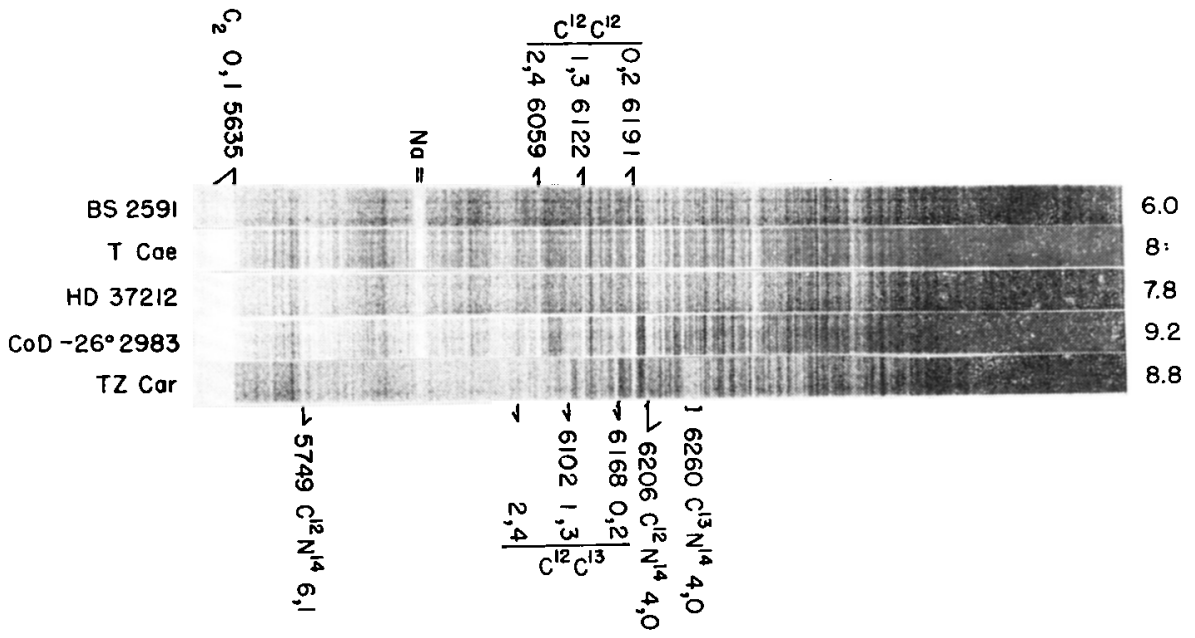

Fig. 2. Typical carbon stars photographed at $106 \AA \mathrm{mm}^{-1}$ with the 60 -in. reflector at Cerro Toloro Inter-American Observatory on Eastman 098-02 spectrographic plates.

Another rather obvious conclusion from Figure 3 is that when $\mathrm{CN}$ is fairly abundant there are no windows in the red region where a real band-free continuum can be observed. As long as only $\mathrm{C}^{12} \mathrm{~N}^{14}$ is present the main heads at $5749 \AA$ could be used as fairly good measures of total $\mathrm{CN}$ abundance, but as soon as the amount of $\mathrm{C}^{13} \mathrm{~N}^{14}$ becomes appreciable the heads are so much smoothed that any photometric measurements, including those of spectrophotometric scanners or narrow-band photometry, would very much underestimate the abundance of $\mathrm{CN}$. The only way to avoid the effect is to use only $\Delta v=0$ heads, for which vibrational isotopic shifts are not appreciable, but such bands are not available in the visual and near infrared regions.

The effect of general observation of features by both isotopic cyanogen molecules is roughly just as serious in the photographic infrared as in the red region shown in Figure 2. For the $\Delta v=+2$ sequence the isotopic shift remains between 40 and $45 \AA$, 


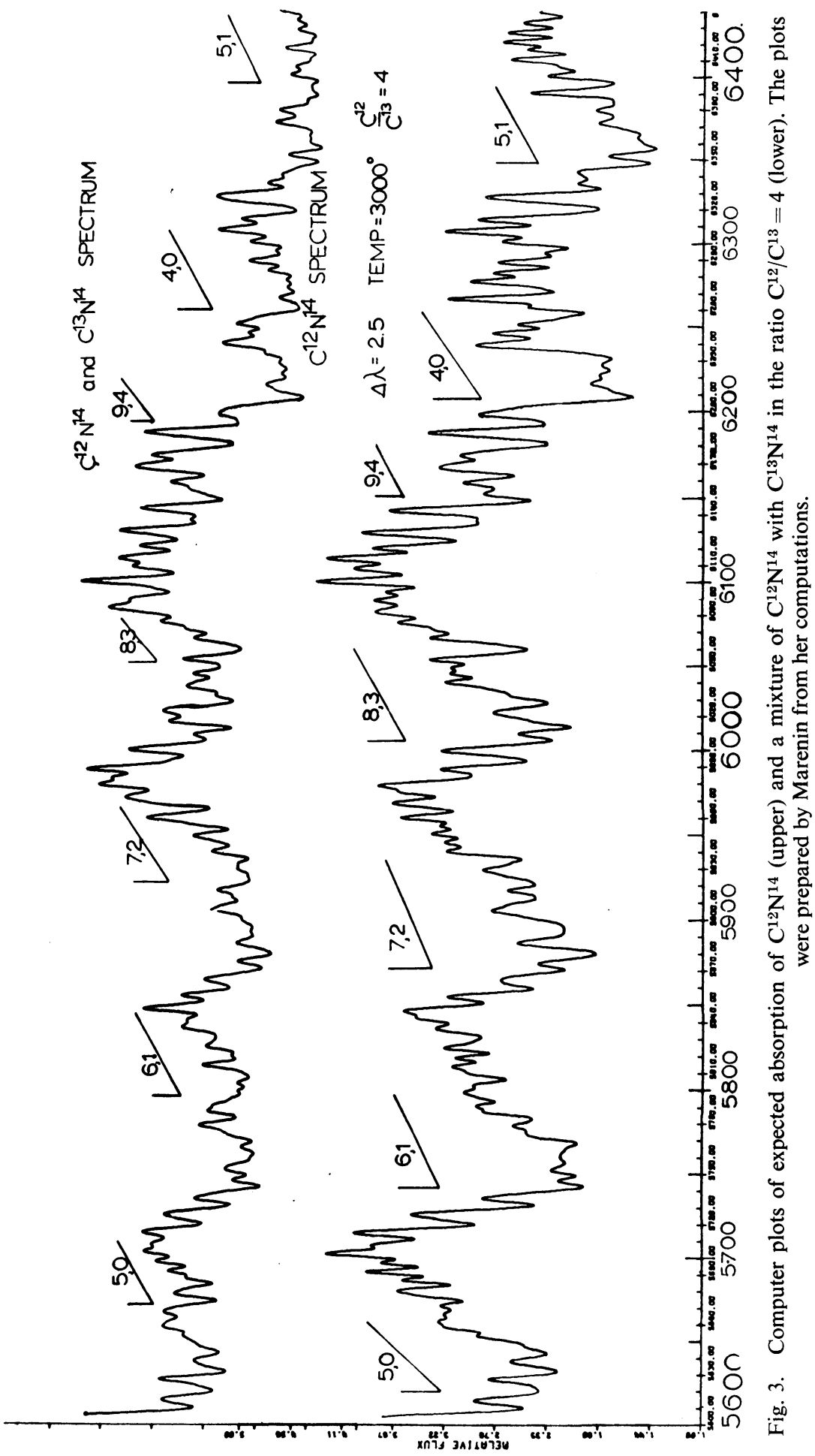


almost as great as for the red bands. Only the first of the $\lambda 7800$ group (at $7850 \AA$ ) of $\mathrm{C}^{12} \mathrm{~N}^{14}$ bands will be reasonably free from contamination by $\mathrm{C}^{13} \mathrm{~N}^{14}$. For hundreds of angstroms redward from there nearly all other features, atomic and molecular, will be subject to heavy blanketing, which increases with both the $C^{13} / C^{12}$ ratio and the total $\mathrm{CN}$ concentration.

The group of stars intermediate between the C-stars and the S-stars are particularly difficult to classify on any system that will be consistent with the types assigned to more heavily banded spectra. Figure 4 shows two of the more striking southern stars in this group. These are UY Cen, studied by Feast (1968) and Catchpole (1971) at Radcliffe, and the red star which was found to be strongly variable by Welch in New Zealand. It was actually noticed by Henize some years ago, and classified as a member of the intermediate group by Feast before I photographed it last winter, without knowing of their work. It is one of the most spectacularly red stars that I have seen through a telescope, and is almost certainly a Mira variable, although the strong $\mathrm{H} \alpha$ emission on this plate is burned out in the enlargement.

That stars like these form a subgroup in which $\mathrm{C} \approx 0$ has come to be generally accepted through the work of Bidelman, Feast, and particularly Stephenson (1967), who formally defined them as a group called CS stars. Recently, Feast and I have thought it might be useful to split them into two groups, calling them CS stars if $\mathrm{C}_{2}$ bands were visible, implying $\mathrm{O} / \mathrm{C}<1$, and $\mathrm{SC}$ stars if $\mathrm{ZrO}$ bands are present, indicating $\mathrm{O} / \mathrm{C}>1$. Most of these stars vary appreciably in light (and temperature), and even if no bands (except the ubiquitous $\mathrm{CN}$ ) are visible at maximum light, they are likely to exhibit the defining bands weakly when near minimum light. Actually, the same suggestion was made at least two years ago by Dolidze (1969). Admittedly, the question of notation would then prove embarrassing if we find stars with such exactly equal amounts of oxygen and carbon (within perhaps $\frac{1}{2} \%$ ), that neither set of bands ever appears. Such stars must be extremely rare, however, and can always be classified as peculiar! One possible star of this kind is CY Cyg, which is being analyzed by Culver (1971). Unfortunately, it is nearly constant in light, and its excitation temperature of $2600-2800 \mathrm{~K}$ is a little too high for a definite test.

Along with these comparatively rare CS and SC stars there are a considerable number of carbon stars that have somewhat stronger $C_{2}$ bands but show essentially the same characteristics, particularly the very strong atomic lines. The D-lines of sodium in Figure 4 are a striking example, and in such stars as WZ Cas they have total absorptions as great as $8 \mathrm{~A}$. In the original $\mathrm{C}$-system we used the $\mathrm{D}$-lines as one of the indicators of temperature, but it can scarcely be doubted now that they are enormously influenced by overlying molecular opacity. There may also be appreciable differences in the abundance of sodium itself. I am afraid that the net result is that we can put very little confidence in the D-lines as temperature parameters. The potassium resonance lines in the near infrared ( $\lambda \lambda 7665$ and 7699$)$ are more reliable, but they also show some anomalies connected with opacity, abundance, and luminosity.

I do not mean to be unduly pessimistic about the prospects for better classification of the carbon stars. All spectral classification can be thought of as essentially component 
analysis carried out either visually or by measurement and automatic reduction. For the carbon stars we must just face the fact that there are more variables to take into account. This probably means that in establishing standards we must combine the information from several spectral regions. If we had data from the far red and infrared regions comparable to the valuable set of intensities of blue and yellow lines and bands compiled by Yamashita (1967), considerable progress would be possible.

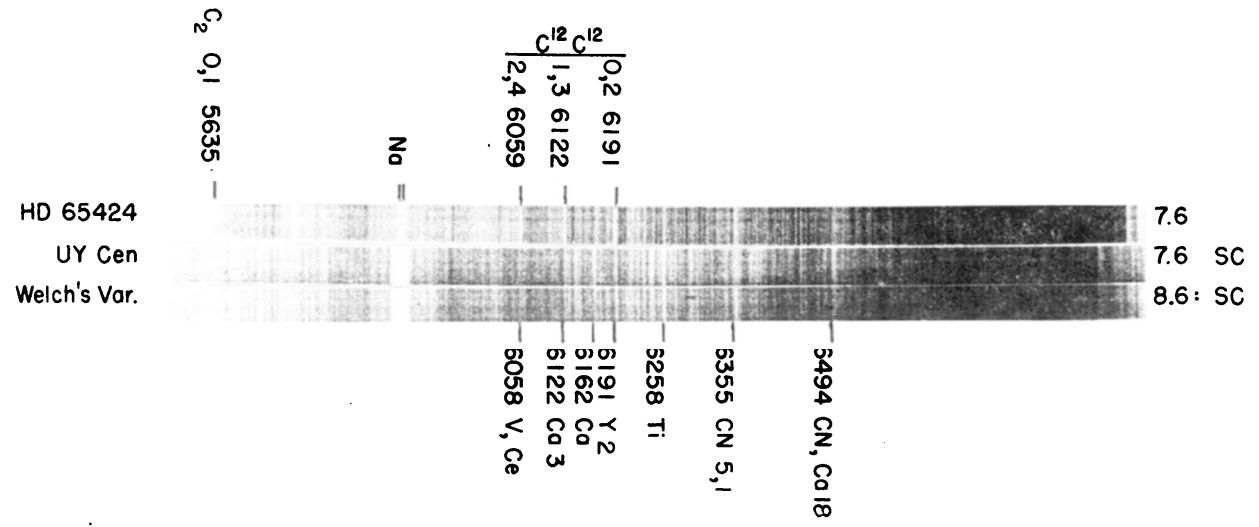

Fig. 4. Spectrograms of two southern SC stars compared with the typical middle carbon star HD 65424. Same scale and source as Figure 2.

Simultaneous photometric data on the blue-violet depression to augment the valuable visual estimates made originally by Shane (1928) would also be valuable. This depression is apparently due at least in part to the $\mathrm{C}_{3}$ molecule, and dissociation curves (cf. Tsuji, 1964) suggest that the abundance of this molecule increases so rapidly with falling temperatures (wherever carbon is abundant), that the appearance or disappearance of these bands may serve to define a rather critical temperature.

\section{References}

Bidelman, W. P.: 1953, Astrophys. J. 117, 25.

Bouigue, R.: 1954, Ann. Astrophys. 17, 104.

Catchpole, R. M.: 1971, Not yet published.

Culver, R.: 1971, Ph.D. Thesis, Ohio State Univ.

Dolidze, M. R.: 1969, Astron. Tsirkular, No. 507, 6; No. 514, 4.

Feast, M. W.: 1968, in L. Perek (ed.), Highlights of Astronomy, D. Reidel Publ. Co., Dordrecht, Holland. p. 237.

Gordon, R. C. P.: 1968, Astrophys. J. 153, 915.

Gordon, R. C. P.: 1971, Publ. Astron. Soc. Pacific 83, 667.

Greene, A. 1971, Ph.D. Thesis, Ohio State Univ.

Griffin, R. F. and Redman, R. O.: 1960, Monthly Notices Roy. Astron. Soc. 120, 287.

Janes, K. A. and McClure, R. D.: 1971, Astrophys. 165, 561.

Keenan, P. C. and Morgan, W. W.: 1941, Astrophys. J. 94, 501.

Kholopov, A. Z.: 1971, Astron. Tsirkular, No. 601, 6.

McClure, R. D.: 1970, Astron. J. 75, 41.

McKellar, A.: 1949, Publ. Dominion Astrophys. Obs., Victoria 7, №. 26, 395. 
Marenin, I. and Greene, A.: 1971, Bull. Am. Astron. Soc. 3, 379.

Merrill, P. W. 1956, Publ. Astron. Soc. Pacific 68, 70.

Morgan, W. W. and Abt, H.: 1971, Astron. J. 77, 35.

Peery, B. F., Jr.: 1971, Astrophys. J. Letters 163, L1.

Richer, H. B.: 1971, Astrophys. J. 167, 521.

Schadee, A.: 1968, Astrophys. J. 151, 239.

Schmitt, J. L.: 1971, Astrophys. J. 163, 75.

Shane, C. D.: 1928, Lick Obs. Bull. 13, No. 396, 123.

Spinrad, H. and Taylor, B. J.: 1969, Astrophys. J. 157, 1279.

Stephenson, C. B.: 1967, Astrophys. J. 150, 543.

Torres-Peimbert, S. and Wallerstein, G.: 1966, Astrophys. J. 146, 724.

Tsuji, T.: 1964, Tokyo Obs. Annals 9, No. 1.

Williams, P. M.: 1971, Observatory 91, 37.

Wurm, K.: 1940, Himmelswelt 50, 20.

Yamashita, Y.: 1967, Publ. Dominion Astrophys. Obs., Victoria 13, No. 5, 67. 